\title{
Prevalence of and Risk Factors for Multidrug-Resistant Bacteria in Urine Cultures of Spinal Cord Injury Patients
}

\author{
Min-Soo Kang, MD, Bum-Suk Lee, MD, Hye-Jin Lee, MD, Seung-Won Hwang, MD, Zee-A Han, MD, PhD
}

Department of Physical Medicine and Rehabilitation, National Rehabilitation Center, Seoul, Korea

\begin{abstract}
Objective To identify the prevalence of multidrug-resistant (MDR) bacteria and identify their specific risk factors in routine urine specimens of spinal cord injury (SCI) patients.

Methods This study was designed in a retrospective manner, reviewing the medical records of SCI patients who were admitted to a specialized SCI unit between January 2001 and December 2013. Patients were investigated for age, gender, American Spinal Injury Association impairment scale, SCI level, duration after injury, bladder management method, and hospitalization history within four weeks prior to visiting our unit. The results of routine urine cultures including presence of MDR organisms were analyzed.

Results Among the total 2,629 urine samples from the newly admitted SCI patients, significant bacteriuria was identified in 1,929 (73.4\%), and MDR organisms were isolated in $29(1.1 \%)$ cultures. There was an increasing trend of MDR organism prevalence from 2001 to $2013(\mathrm{p}<0.01)$. The isolation of MDR organisms in inpatients who were admitted for rehabilitation ( $1.3 \%)$ was significantly higher than it was among community-residing persons $(0.2 \%)$ $(\mathrm{p}<0.05)$. By voiding method, patients who used a suprapubic indwelling catheter $(3.3 \%)$ or a urethral indwelling catheter $(2.6 \%)$ showed a higher rate of MDR organism isolation $(\mathrm{p}<0.05)$.

Conclusion There was an increasing trend of MDR organism isolation in SCI patients. Inpatients and persons who used indwelling catheters showed a higher risk of MDR organism isolation.
\end{abstract}

Keywords Spinal cord injuries, Bacteriuria, Urinary tract infections, Multiple drug resistance

Received March 30, 2015; Accepted May 11, 2015

Corresponding author: Zee-A Han

Department of Physical Medicine and Rehabilitation, National Rehabilitation Center, 58 Samgaksan-ro, Gangbuk-gu, Seoul 01022, Korea

Tel: +82-2-901-1802, Fax:+82-2-901-1591, E-mail: zeeahan@catholic.ac.kr

(c) This is an open-access article distributed under the terms of the Creative Commons Attribution Non-Commercial License (http://creativecommons. org/licenses/by-nc/4.0) which permits unrestricted noncommercial use, distribution, and reproduction in any medium, provided the original work is properly cited.

Copyright $\odot 2015$ by Korean Academy of Rehabilitation Medicine

\section{INTRODUCTION}

Many patients with spinal cord injury (SCI) have concomitant neurogenic bladder [1]. Because of impaired bladder storage, voiding dysfunction, and use of indwelling catheters after SCIs, these patients often experience urinary tract infections (UTIs) [2,3]. Repeated UTIs can cause upper urinary tract deterioration, and related chronic or repeated exposure to the antibiotics used to treat UTI increases the risk of multidrug-resistant (MDR) bacteria colonization $[4,5]$. Resistance in UTI-causing 
bacterial strains in SCI patients has been increasing [6]. Therefore, it is important to determine the prevalence of MDR urinary tract organisms to determine appropriate UTI treatment in SCI patients who are admitted to rehabilitation hospitals. In addition, continuous and longterm surveillance of MDR prevalence to identify changes in yearly trends is important for infection control in SCI patients.

In 2011, the Infectious Disease Control and Prevention Act in the Republic of Korea designated six bacterial strains-vancomycin-resistant Staphylococcus aureus (VRSA), vancomycin-resistant Enterococci (VRE), methicillin-resistant $S$. aureus (MRSA), multidrug-resistant Pseudomonas aeruginosa (MRPA), multidrug-resistant Acinetobacter baumannii (MRAB), and carbapenemresistant Enterobacteriaceae (CRE) - as MDR organisms that require monitoring and management at the national level. Although a number of large-scale reports related to MDR organisms in Korea were published as a result, relatively few specific reports focused on particular diseases or cohorts. In addition, no domestic report exists to date on the prevalence of urinary tract MDR organisms in SCI patients or on factors that contribute to colonization by MDR organisms in SCI patients.

The objective of this study was to analyze clinical and laboratory data collected from SCI patients who had been admitted to a freestanding rehabilitation hospital from 2001 to 2013 in order to determine the prevalence of MDR organisms in routine urine specimens and identify factors that increase their occurrence.

\section{MATERIALS AND METHODS}

\section{Subjects}

The subjects in this study were patients with SCI who were admitted to the specialized SCI unit of a freestanding rehabilitation hospital from January 2001 to December 2013. For the subset of patients who had been hospitalized more than once, only data from their first admission were included in the analyses. Patients with incomplete medical records or those who did not undergo initial routine urine culture were excluded from the study.

\section{Data collection}

Patient information from hospitalization records was investigated retrospectively. Age, gender, American Spi- nal Injury Association (ASIA) impairment scale, SCI level, time since injury, hospitalization history at other institutions within the previous four weeks, and voiding methods of patients with SCI at the time of admission were determined by examining medical records. To establish hospitalization history, we determined if patients had been admitted to any other hospitals less than 30 days before admission to our hospital. In this study, voiding methods were categorized into four types: catheter-free, intermittent catheterization, urethral indwelling catheter, or suprapubic indwelling catheter. The catheter-free voiding methods included reflex voiding and incontinence.

Among the 3,199 SCI patients who were admitted to the hospital between 2001 and 2013, a total of 2,629 patients were selected for analysis using the aforementioned methods. The subjects consisted of 1,921 men and 708 women with a mean age of $45.58 \pm 15.17$ years. Patient categorization based on the ASIA impairment scale (AIS) showed that AIS category A was most common (45.4\%). Among levels of spinal cord injury, tetraplegia (50.8\%) was slightly more common than paraplegia $(49.2 \%)$. The average length of disease after spinal cord injury was $33.81 \pm 62.23$ months. Among the study subjects, $80.2 \%$ had a hospitalization history. Intermittent catheterization $(44.1 \%)$ was the most common bladder management method, followed by catheter-free (33.6\%), urethral indwelling catheter (18.8\%), and suprapubic indwelling catheter (3.5\%) (Table 1).

\section{Laboratory methods}

The study hospital performs routine urine cultures on all admitted patients regardless of the presence or absence of symptoms at the time of admission. The hospital utilizes the clean-catch technique for patients who are capable of spontaneous voiding and catheterization for those who are not. For sample collection from patients who were not capable of spontaneous voiding and who had an indwelling catheter, aseptic aspiration was performed with a sterile syringe in a clamped state after catheter change. For patients with intermittent catheterization, samples were collected during the procedure. The samples were cultured for bacteria in a routine laboratory, and the culture test results were reported, including the presence of MDR bacterial strains.

In this study, we investigated the results of single routine urine cultures conducted at the time of admission as 
Table 1. Demographics and clinical variables $(n=2,629)$

\begin{tabular}{|c|c|}
\hline Variable & Value \\
\hline \multicolumn{2}{|l|}{ Gender } \\
\hline Male & $1,921(73.1)$ \\
\hline Female & $708(26.9)$ \\
\hline \multicolumn{2}{|l|}{ Hospitalization history } \\
\hline Hospitalized at another institute & $2,109(80.2)$ \\
\hline Community-resided & $520(19.8)$ \\
\hline \multicolumn{2}{|l|}{ Neurological level } \\
\hline Tetraplegia & $1,335(50.8)$ \\
\hline Paraplegia & $1,294(49.2)$ \\
\hline \multicolumn{2}{|l|}{ AIS } \\
\hline A & $1,193(45.4)$ \\
\hline B & $398(15.1)$ \\
\hline $\mathrm{C}$ & $453(17.2)$ \\
\hline $\mathrm{D}$ & $585(22.3)$ \\
\hline Age (yr) & $45.58 \pm 15.17$ \\
\hline$<20$ & $67(2.5)$ \\
\hline $20-29$ & $346(13.2)$ \\
\hline $30-39$ & $604(23.0)$ \\
\hline $40-49$ & $580(22.1)$ \\
\hline $50-59$ & $507(19.3)$ \\
\hline $60-69$ & $338(12.8)$ \\
\hline$\geq 70$ & $187(7.1)$ \\
\hline Mean time since injury (mo) & $33.81 \pm 62.23$ \\
\hline$<3$ & $472(17.9)$ \\
\hline $3-12$ & $996(37.9)$ \\
\hline$>12$ & $1,161(44.2)$ \\
\hline \multicolumn{2}{|l|}{ Voiding method } \\
\hline Catheter-free & $883(33.6)$ \\
\hline Intermittent catheterization & $1,161(44.1)$ \\
\hline Urethral indwelling catheter & $494(18.8)$ \\
\hline Suprapubic indwelling catheter & $91(3.5)$ \\
\hline
\end{tabular}

Values are presented as number (\%) or mean \pm standard deviation.

AIS, American Spinal Injury Association impairment scale.

well as the incidence of MDR isolation.

\section{Definitions of antimicrobial resistance}

In this study, the following definitions were used for analyses of urine culture results. First, $10^{5}$ colony-forming units (CFU)/mL or higher was defined as significant bacteriuria [7]. The generally accepted criteria for significant bacteriuria for SCI patients were set by the National Institute on Disability and Rehabilitation Research and vary depending on voiding method [8]. However, in this study, significant bacteriuria was uniformly defined as $10^{5} \mathrm{CFU} /$ $\mathrm{mL}$ regardless of voiding method because the hospital microbiology laboratory did not provide data when the count was below 1,000 colonies $/ \mathrm{mL}$. This criterion has been utilized in many previous SCI patient studies [7,9]. Second, polymicrobial bacteriuria was defined as the detection of two or more bacterial species in a single specimen culture [10]. Finally, MDR bacteria detection was defined as identification of one of six strains, VRSA, VRE, MRSA, MRPA, MRAB, or CRE, in the urine culture. These six MDR bacteria were designated by the Infectious Disease Control and Prevention Act in January 2011 as MDR organisms that required monitoring.

\section{Data analysis}

Statistical analysis was conducted using SPSS ver. 20.0 (IBM, Armonk, NY, USA). The prevalence of significant bacteriuria, polymicrobial bacteriuria, and MDR organisms was determined, and the corresponding urine test results were categorized based on age, gender, AIS, SCI level, time since injury, voiding method, and history of hospitalization at other institutions. Chi-square tests were used to verify statistically significant differences in significant bacteriuria as well as prevalence of polymicrobial bacteriuria, bacterial species, and MDR organisms according to gender, hospitalization history, and voiding method. To estimate the risk factors that are thought to influence by MDR organism colonization, the odds ratios for age, gender, admission year, AIS, SCI level, time since injury, voiding method, and history of hospitalization were determined using multivariate logistic regression analysis to control for potential confounders. Statistical significance was defined as $\mathrm{p}<0.05$.

\section{RESULTS}

\section{Urine cultures}

\section{Significant bacteriuria}

Of the 2,629 urine cultures, 1,929 (73.4\%) had significant bacteriuria (Table 2), particularly, more women (78.4\%) than men (71.5\%). A higher percentage of individuals with urethral $(84.8 \%)$ or suprapubic $(90.1 \%)$ indwelling catheters showed significant bacteriuria compared with 


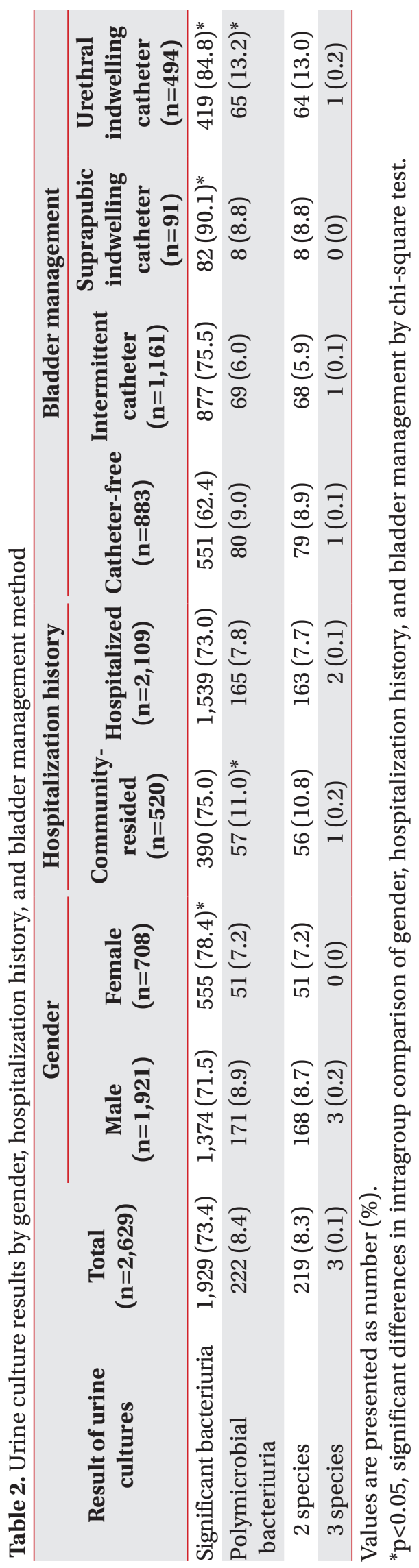

those without.

\section{Polymicrobial bacteriuria}

Polymicrobial bacteriuria was detected in 222 (8.4\%) urine cultures (Table 2), including a higher percentage of individuals who lived in communities $(11.0 \%)$ than that for patients with a hospitalization history (7.8\%). A higher percentage of individuals with urethral indwelling catheters $(13.2 \%)$ were positive for polymicrobial bacteriuria than were those without. Polymicrobial bacteriuria with two and three species was detected in 219 (8.3\%) and three $(0.1 \%)$ urine cultures, respectively.

\section{Bacterial species}

Including the polymicrobial samples, a total of 2,154 bacterial isolates were identified from 1,929 culture-positive urine samples (Table 3). Among these 2,154 isolates, $1,784(82.8 \%)$ were gram-negative bacteria, including Escherichia coli (628, 29.2\%), Klebsiella spp. (330, 15.3\%), and Pseudomonas spp. (180, 8.4\%). The most frequently identified strain, E. coli, was also more frequently isolated in women than men $(\mathrm{p}<0.01)$. By history of hospitalization in other institutions, E. coli was less frequently isolated in those with a hospitalization history than in those without $(\mathrm{p}<0.01)$. Differences in $E$. coli isolation frequency were also detected for different bladder management methods: samples obtained from patients with indwelling catheters had a lower $E$. coli isolation frequency $(\mathrm{p}<0.01)$. Klebsiella was the second most frequently detected strain after $E$. coli. Its prevalence also varied according to bladder management method ( $\mathrm{p}<0.01)$, with greater frequency in patients who underwent intermittent catheterization. Pseudomonas was more commonly isolated in men than in women $(\mathrm{p}<0.01)$. Among bladder management methods, Pseudomonas was more commonly identified in those with suprapubic or urethral indwelling catheters $(\mathrm{p}<0.01)$. Acinetobacter was more commonly isolated in male than female subjects $(\mathrm{p}<0.01)$. We also detected differences in its prevalence depending on voiding method, with catheter-free individuals having the highest isolation rate $(\mathrm{p}<0.05)$. However, gram-positive bacteria were isolated in the $348(16.2 \%)$ cases. The major gram-positive bacteria strains were Enterococcus spp. $(255,11.8 \%)$ and $S$. aureus $(43,2.0 \%)$. Between these, Enterococcus was most frequently isolated gram-positive bacteria. Enterococcus was also more commonly iso- 


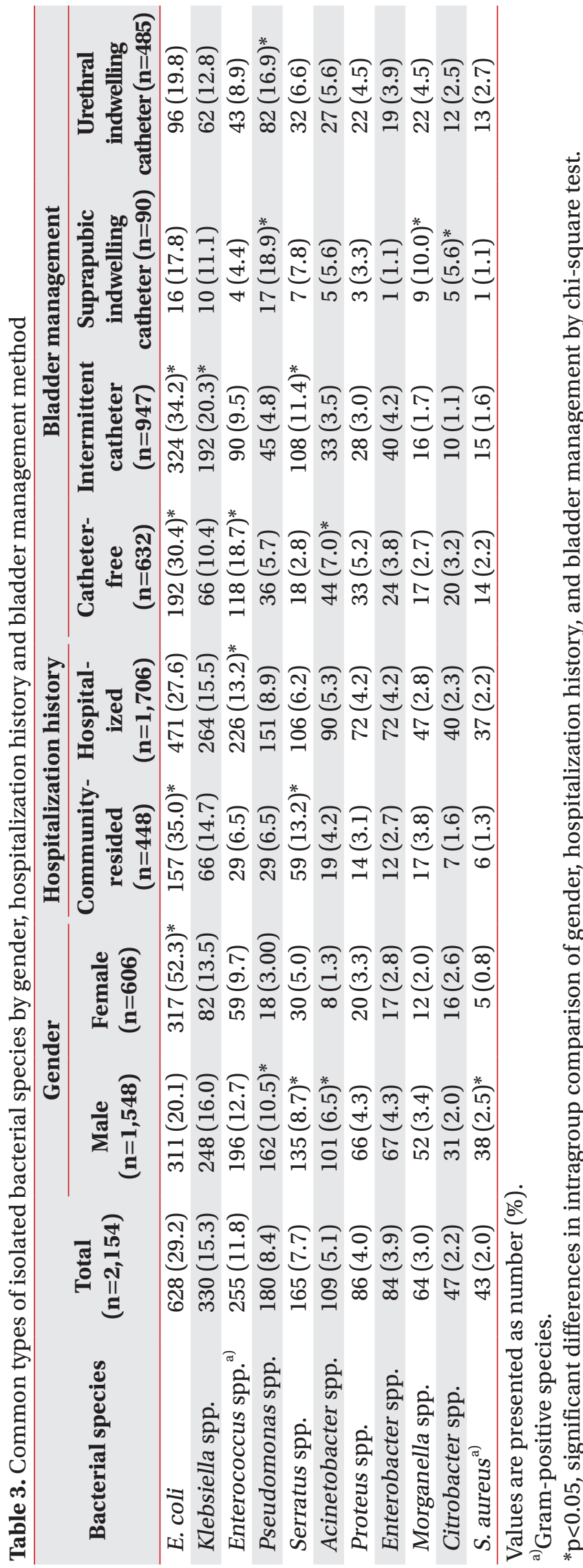

lated in individuals with hospitalization history in other institutions than in those without $(\mathrm{p}<0.01)$. In addition, identification rates differed based on voiding method, with the highest rate in catheter-free individuals $(\mathrm{p}<0.01)$. $S$. aureus was identified 43 times $(2.0 \%)$ and more commonly in men than in women $(\mathrm{p}<0.05)$.

Table 4. MDR isolation by specific variables

\begin{tabular}{|c|c|}
\hline $\begin{array}{r}\text { Variable } \\
\end{array}$ & MDR isolation \\
\hline Total $(\mathrm{n}=2,629)$ & $29(1.1)$ \\
\hline \multicolumn{2}{|l|}{ Gender } \\
\hline Male & $26(1.4)^{*}$ \\
\hline Female & $3(0.4)$ \\
\hline \multicolumn{2}{|l|}{ Hospitalization history } \\
\hline Hospitalized at another institute & $28(1.3)^{*}$ \\
\hline Community-resided & $1(0.2)$ \\
\hline \multicolumn{2}{|l|}{ Neurological level } \\
\hline Tetraplegia & $22(1.6)^{*}$ \\
\hline Paraplegia & $7(0.5)$ \\
\hline \multicolumn{2}{|l|}{ AIS } \\
\hline A & $13(1.1)$ \\
\hline B & $5(1.3)$ \\
\hline $\mathrm{C}$ & $7(1.5)$ \\
\hline $\mathrm{D}$ & $4(0.7)$ \\
\hline \multicolumn{2}{|l|}{ Age (yr) } \\
\hline$<20$ & $0(0.0)$ \\
\hline $20-29$ & $5(1.4)$ \\
\hline $30-39$ & $4(0.7)$ \\
\hline $40-49$ & $8(1.4)$ \\
\hline $50-59$ & $3(0.6)$ \\
\hline $60-69$ & $6(1.8)$ \\
\hline$\geq 70$ & $3(1.6)$ \\
\hline \multicolumn{2}{|l|}{ Mean time since injury (mo) } \\
\hline$<3$ & $13(2.8)^{*}$ \\
\hline $3-12$ & $11(1.1)$ \\
\hline$>12$ & $5(0.4)$ \\
\hline \multicolumn{2}{|l|}{ Voiding method } \\
\hline Catheter-free & $5(0.6)$ \\
\hline Intermittent catheterization & $8(0.7)$ \\
\hline Urethral indwelling catheter & $13(2.6)^{*}$ \\
\hline Suprapubic indwelling catheter & $3(3.3)^{*}$ \\
\hline
\end{tabular}

Values are presented as number (\%).

MDR, multidrug-resistant; AIS, American Spinal Injury Association impairment scale.

${ }^{*} \mathrm{p}<0.05$, significant differences in intragroup comparison of each specific variable by chi-square test. 


\section{MDR organisms}

MDR organisms were detected in 29 (1.1\%) of the 2,629 urine samples (Table 4). MDR isolates were not detected from 2001 to 2004; they were first isolated in 2005 and have been detected every year since. Furthermore, whereas three (1.3\%) MDR isolates were reported in 2005, eight (4.7\%) MDR species were isolated in 2013, the highest frequency of MDR organism detection to date (Fig. 1). The identified MDR organisms included MRSA (18), MRAB (6), VRE (3), and MRPA (2) (Fig. 2), and MRSA was most frequently observed (62.1\%). Among voiding methods, the MDR prevalence was highest in patients with urethral $(2.6 \%)$ or suprapubic (3.3\%) indwelling catheters compared with those without (Table 4 ). Those with a history of hospitalization (1.3\%) also experienced significantly more frequent MDR organism infections compared with those without $(0.2 \%)$. Of the 29 patients who

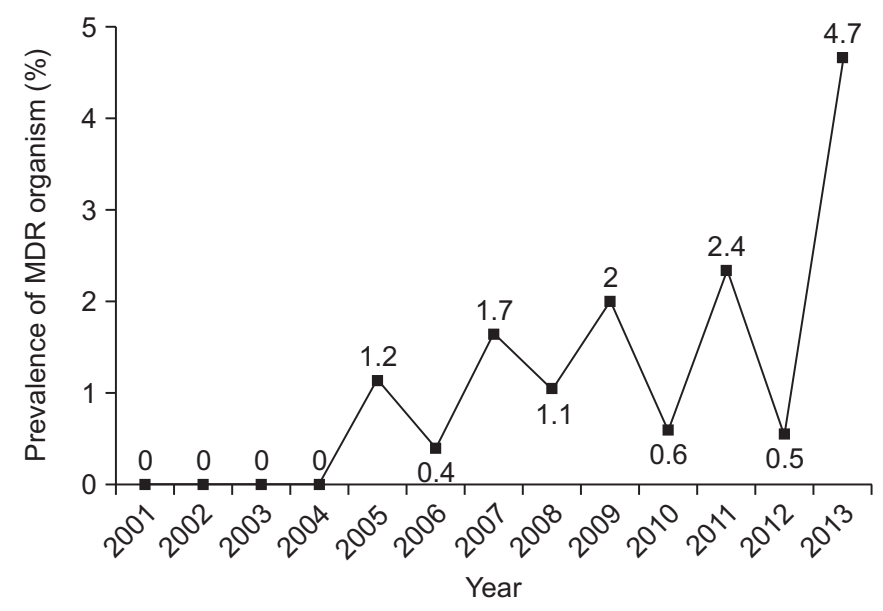

Fig. 1. Yearly prevalence of multidrug-resistant (MDR) organism isolation from 2001 to 2013. were positive for MDR organisms, most had a history of hospitalization within four weeks of admission; only one patient who was MRSA-positive did not have a history of hospitalization (Table 4). Men (1.4\%) had greater MDR organism isolation frequency than did women $(0.4 \%)$ $(\mathrm{p}<0.05)$. Analysis according to injury level revealed a higher MDR organism isolation frequency in tetraplegic $(1.6 \%)$ than paraplegic $(0.5 \%)$ patients $(\mathrm{p}<0.01)$. MDR organism isolation frequency was also greater in patients whose time since injury was less than three months $(2.8 \%)$ $(p<0.01)$. There were no significant differences in the frequency of MDR organism isolation based on age or AIS (Table 4).

\section{Risk factors for MDR organism isolation}

\section{Demographic factors}

After confounders were controlled for in the logistic regression analysis, MDR organism occurrence differed

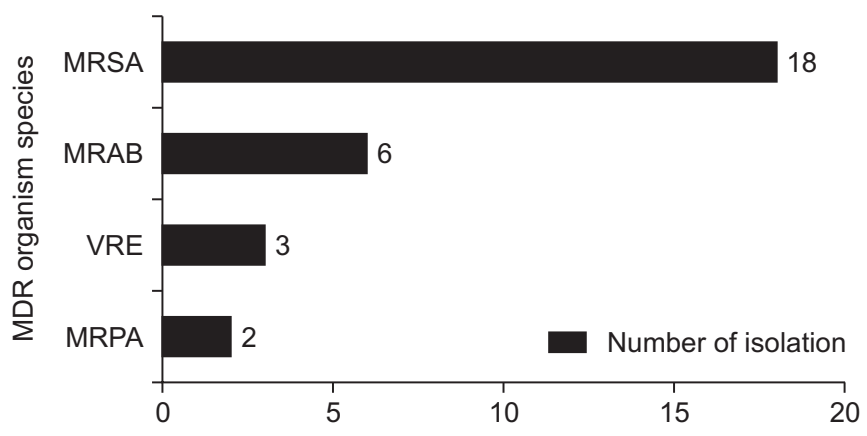

Fig. 2. Species of cultured multidrug-resistant (MDR) isolates. MRSA, methicillin-resistant Staphylococcus aureus; MRAB, multidrug-resistant Acinetobacter baumannii; VRE, vancomycin-resistant Enterococci; MRPA, multidrug-resistant Pseudomonas aeruginosa.

Table 5. Risk factors associated with MDR organism isolation

\begin{tabular}{lcc}
\hline \multicolumn{1}{c}{ Predictive factor } & OR (95\% CI) & p-value* \\
\hline Sex (male) & $2.881(0.854-9.720)$ & 0.088 \\
Year at admission (from 2001 to 2013) & $1.249(1.103-1.414)$ & $<0.001$ \\
Tetraplegia & $2.120(0.878-5.119)$ & 0.095 \\
Hospitalization history & $4.330(0.574-32.661)$ & 0.155 \\
Catheter-free & (Reference) & - \\
\hline Intermittent catheterization & $1.453(0.467-4.521)$ & 0.519 \\
\hline Suprapubic indwelling catheter & $3.335(0.763-14.571)$ & 0.109 \\
\hline Urethral indwelling catheter & $3.547(1.246-10.097)$ & 0.018 \\
\hline
\end{tabular}

MDR, multidrug-resistant; OR, odds ratio; CI, confidence interval.

${ }^{*}$ Multivariate logistic regression analysis. 
significantly depending on admission year $(\mathrm{p}<0.01)$; MDR occurrence risk also increased annually by 1.249 from 2001 to 2013 (Table 5). By gender, men had 2.881 times the risk of that for women, a finding with borderline statistical significance $(0.05<\mathrm{p}<0.1)$. For those with hospitalization history in other institutions, MDR risk as represented by odds ratio was 4.330 times greater than for those without, although this difference was not statistically significant; this observation should be carefully considered. Age was not significantly correlated with risk of MDR isolation.

\section{SCI-associated factors}

Tetraplegic SCI patients had 2.120 times the risk of being positive for MDR isolates than did paraplegic patients, a finding with borderline statistical significance $(0.05<\mathrm{p}<0.1)$. Time since injury and AIS were not significantly correlated with the risk of MDR isolates (Table 5).

\section{Bladder management method-associated factors}

Patients with urethral indwelling catheters had 3.547 times the MDR organism isolation rate of that in patients who used catheter-free voiding, a statistically significant difference $(\mathrm{p}<0.05)$. Similarly, patients with suprapubic indwelling catheters had 3.335 times the MDR organism isolation frequency of that in patients who used catheterfree voiding. Although this difference was not statistically significant, its odds ratio was high (Table 5).

\section{DISCUSSION}

This study analyzed the results of routine urine cultures from over 2,600 SCI patients who were admitted to a single institution over the last 13 years. The study strengths included detailed urine culture results based on extensive data from SCI patients and the fact that various confounders were controlled for in the analysis of risk factors that influenced the incidence of MDR organisms. Previous studies on urine culture in SCI patients that were conducted in Korea were of smaller scale and had a number of limitations. This study provides a sound basis for looking at urinary bacterial colonization and MDR organism occurrence in domestic SCI patients.

Bacterial colonization of the urinary tract in SCI patients generally occurs because of the spread of gramnegative bacteria that reside in the bowel, perineum, or urethra following insertion of instruments into the bladder, contamination from an environmental source, or cross-contamination from other patients [10-12]. Individuals who have experienced urinary tract colonization have increased risks of further tissue invasion and recurrent symptomatic UTI [10]. In this study, 1,929 (73.4\%) of 2,629 SCI patients were positive for significant bacteriuria based on routine urologic examination of urine cultures. This result was similar to those in previous studies that reported $70 \%-87.3 \%$ bacterial culture positivity in urine cultures from SCI patients $[9,13,14]$. However, previous studies have also reported that $63.3 \%$ of patients with symptomatic UTIs showed changes in organisms compared with their initial urine cultures [15]. Therefore, it is important to compare urine test results taken when the patient presents with clinical symptoms of UTI and at the time of admission.

Polymicrobial growth in urine cultures from individuals with functionally normal urinary tracts is typically attributed to contaminated specimens, and urine culture is often repeated. However, detection of polymicrobial growth in urine cultures from SCI patients and urinary symptoms is highly likely to have clinical significance [16]. In this study, we analyzed urine culture tests that were conducted regardless of presence of symptoms in SCI patients, and polymicrobial bacteriuria was detected in $8.4 \%$ of cases. It is likely that the frequency would have been even higher among SCI patients with urinary symptoms. Furthermore, polymicrobial bacteriuria is reportedly related to increased antibiotic resistance [17].

The bacterial species detected in this study, in order of frequency, included E. coli, Klebsiella, Enterococcus, and Pseudomonas. Because Enterococcus comprises VRE, Pseudomonas comprises MRPA, Acinetobacter comprises MRPA, and $S$. aureus comprises MRSA, the prevalence of these bacterial species should be watched carefully. Some had different detection frequencies according to patient gender, hospitalization history, and bladder management method, findings similar to those of previous studies [18].

This study showed that there has been a significant increase in the prevalence of MDR organisms in the last 13 years. In particular, the detection rate in 2013 was high, at $4.7 \%$. This is a global trend that is often attributed to increased antibiotic usage. Domestically, increased detection of MDR organisms is recognized as a significant 
problem in individual hospitals.

According to the analysis results of healthcare-associated infectious disease monitoring data published by the Infectious Disease Monitor Department of Center for Disease Control in the Republic of Korea, MDR organisms reported by 100 domestic sample-monitoring institutions included MRAB $(21,315)$, MRSA $(12,581)$, MRPA $(6,783)$, VRE $(2,515)$, and CRE (977) from January 1 to December 31,2012 , and MRSA $(42,422)$, MRAB $(22,489)$, VRE $(8,280)$, MRPA $(5,914)$ and CRE $(1,839)$ from January 1 to December 31, 2013. Noteworthy changes in 2013 compared with 2012 included a significant increase in MRSA, exceeding MRAB. Although these results were obtained from diverse patients and samples, they can be compared with the results of the current study. In this study, 18 cases of MRSA, six cases of MRAB, three cases of VRE, and two cases of MRPA were detected in the urine cultures of SCI patients. Based on the order of frequency for each MDR organism, the observed trends were generally similar to the 2013 data from the Center for Disease Control, except for CRE detection.

In an acute hospital setting, many SCI patients receive broad-spectrum systemic antibiotic treatment for various reasons, including UTIs. This general usage promotes the colonization of resistant bacteria $[5,10]$ and could explain the higher frequency of MDR organisms in patients with a history of hospitalization. The rationale for defining hospitalization history as having a history of inpatient treatment at another institution within 30 days of admission to the hospital was that symptomatic infections within 30 days of discharge are considered to be nosocomial infections.

Analysis of urine culture test results by voiding method revealed that patients with suprapubic or urethral indwelling catheters had a higher frequency of significant bacteriuria and isolation of MDR organisms compared with catheter-free patients and those with intermittent catheterization. In addition, polymicrobial bacteriuria was most frequently observed in patients with a urethral indwelling catheter. A previous study on SCI patients reported that the frequency of polymicrobial bacteriuria depended on voiding method [13]. Therefore, in order to reduce urinary tract bacterial colonization, including MDR organisms, indwelling catheters are not recommended. However, SCI patients with indwelling catheters may have been already exposed to various urologic problems related to the urinary tract, which may result in overestimating the frequency of MDR organism isolation or other urine culture test results. In addition, because of limitations in the data that were analyzed in this study, neither incontinence voiding nor spontaneous voiding could be distinguished in the medical records and were therefore both categorized as catheter-free.

A previous study identified risk factors associated with increased detection of MDR bacteria in routine urinary tests for SCI patients, including age (less than 45 years), gender (male), and bladder management method (condom or indwelling catheter) [10]. Results from logistic regression analysis in this study showed that bladder management method (urethral indwelling catheter) and time since injury had the most significant impact on the detection of MDR organisms; gender (male), injury level (tetraplegia), hospitalization history (hospitalized), and other bladder management methods (suprapubic indwelling catheter) were identified as additional factors. Male patients had a comparably greater frequency of MDR organism-causing bacterial species such as Pseudomonas, Acinetobacter and S. aureus than did female patients. In addition, it is assumed that tetraplegic patients will have a longer history of antibiotic usage because of pneumonia or UTIs compared with paraplegic patients. Moreover, patients with a hospitalization history are assumed to be more likely to have a history of medical problems or antibiotic usage in other institutions. Considering these results, routine urine cultures would be useful for detecting MDR organisms in SCI patients who are at high risk for MDR organism colonization. Furthermore, in deciding between voiding methods, use of indwelling catheters should be reevaluated because it may promote colonization by MDR organisms.

This study had a number of limitations. First, it was a single-institutional study that consequently included only patients who were admitted to the institution. Second, this was a retrospective study that reviewed patient charts. Third, because the patient database did not provide information on clinical symptoms and signs, symptomatic and asymptomatic bacteriuria could not be identified. Lastly, because of data limitations, history of urinary problems, antibiotic usage, and length of hospitalization prior to admission to this hospital could not be determined.

This study analyzed urine culture results from SCI pa- 
tients in order to determine the prevalence of MDR organisms and identify factors that lead to increased risk of MDR organism. Although this study analyzed large-scale data from more than 2,600 individuals collected over 13 years, the findings may not be representative because it was a single-institution study. This study also did not measure time to UTI occurrence, antibiotic usage and type, patient medical conditions, or history of intense critical unit usage, which all may be related to risk factors for MDR bacteria colonization. Future studies should be designed to address these limitations.

In this study, $73.4 \%$ of 2,629 routine urine cultures from patients with SCI were positive for significant bacteriuria. The prevalence of MDR organisms gradually increased from 2001 to 2013 and was reported in $4.7 \%$ of all patients in 2013. MDR organisms were more frequently observed in male and tetraplegic patients; most of these patients also had a history of hospitalization in other institutions. Among voiding methods, patients with indwelling catheters had a significantly higher incidence of MDR organisms. Based on the results from this study, clinicians should pay attention to infection control in patients with SCI who are at high risk of MDR organism colonization.

\section{CONFLICT OF INTEREST}

No potential conflict of interest relevant to this article was reported.

\section{REFERENCES}

1. Ginsberg D. The epidemiology and pathophysiology of neurogenic bladder. Am J Manag Care 2013;19(10 Suppl):s191-6.

2. Pannek J. Treatment of urinary tract infection in persons with spinal cord injury: guidelines, evidence, and clinical practice. A questionnaire-based survey and review of the literature. J Spinal Cord Med 2011;34:11-5.

3. Cameron AP, Wallner LP, Tate DG, Sarma AV, Rodriguez GM, Clemens JQ. Bladder management after spinal cord injury in the United States 1972 to 2005. J Urol 2010;184:213-7.

4. Fonte N. Urological care of the spinal cord-injured patient. J Wound Ostomy Continence Nurs 2008;35:323-31.

5. Archibald L, Phillips L, Monnet D, McGowan JE Jr, Tenover F, Gaynes R. Antimicrobial resistance in iso- lates from inpatients and outpatients in the United States: increasing importance of the intensive care unit. Clin Infect Dis 1997;24:211-5.

6. Hinkel A, Finke W, Botel U, Gatermann SG, Pannek J. Increasing resistance against antibiotics in bacteria isolated from the lower urinary tract of an outpatient population of spinal cord injury patients. Urol Int 2004;73:143-8.

7. Faarvang KL, Muller P, Lomberg B, Biering-Sorensen F. Screening for bacteriuria in patients with spinal cord lesion: dipstick test, microscopic examination and urine culture. Spinal Cord 2000;38:106-8.

8. Garcia Leoni ME, Esclarin De Ruz A. Management of urinary tract infection in patients with spinal cord injuries. Clin Microbiol Infect 2003;9:780-5.

9. Menon EB, Tan ES. Urinary tract infection in acute spinal cord injury. Singapore Med J 1992;33:359-61.

10. Waites KB, Chen Y, DeVivo MJ, Canupp KC, Moser SA. Antimicrobial resistance in gram-negative bacteria isolated from the urinary tract in community-residing persons with spinal cord injury. Arch Phys Med Rehabil 2000;81:764-9.

11. Montgomerie JZ. Infections in patients with spinal cord injuries. Clin Infect Dis 1997;25:1285-90.

12. Waites KB, Canupp KC, Brookings ES, DeVivo MJ. Effect of oral ciprofloxacin on bacterial flora of perineum, urethra, and lower urinary tract in men with spinal cord injury. J Spinal Cord Med 1999;22:192-8.

13. Ryu KH, Kim YB, Yang SO, Lee JK, Jung TY. Results of urine culture and antimicrobial sensitivity tests according to the voiding method over 10 years in patients with spinal cord injury. Korean J Urol 2011;52:345-9.

14. Dedeic-Ljubovic A, Hukic M. Catheter-related urinary tract infection in patients suffering from spinal cord injuries. Bosn J Basic Med Sci 2009;9:2-9.

15. Shin JC, Yoo JH, Park JW, Park S, Ahn SJ, Park CI. Difference of organism and their antibiotics sensitivity from urine culture in symptomatic urinary tract infection of spinal cord injury patients. J Korean Acad Rehabil Med 2008;32:38-44.

16. Darouiche RO, Priebe M, Clarridge JE. Limited vs full microbiological investigation for the management of symptomatic polymicrobial urinary tract infection in adult spinal cord-injured patients. Spinal Cord 1997;35:534-9. 
17. Croxall G, Weston V, Joseph S, Manning G, Cheetham $\mathrm{P}, \mathrm{McNally} A$. Increased human pathogenic potential of Escherichia coli from polymicrobial urinary tract infections in comparison to isolates from monomicrobial culture samples. J Med Microbiol 2011;60(Pt
1):102-9.

18. Bennett CJ, Young MN, Darrington H. Differences in urinary tract infections in male and female spinal cord injury patients on intermittent catheterization. Paraplegia 1995;33:69-72. 